\title{
PERCEÇÃO DE ATITUDES PARENTAIS E PROBLEMAS DE COMPORTAMENTO NOS ADOLESCENTES
}

\author{
Maria da Luz Vale-Dias \\ Faculdade de Psicologia e de Ciências da Educação \\ Universidade de Coimbra, Portugal \\ valedias@fpce.uc.pt \\ Cláudia Sepanas Berardo \\ Faculdade de Psicologia e de Ciências da Educação \\ Universidade de Coimbra, Portugal
}

Fecha de Recepción: 9 Junio 2019

Fecha de Admisión: 25 Septiembre 2019

\begin{abstract}
RESUMO
Introdução: 0 papel desempenhado pelas atitudes educativas parentais no desenvolvimento e comportamento na adolescência tem sido amplamente suportado, surgindo um interesse pela sua pesquisa não só enquanto fator protetor, mas também como fator de risco para alguns desvios e problemas de saúde mental. A presente investigação foi concebida com 0 intuito de analisar a associação entre as perceções das atitudes parentais e a manifestação de problemas de comportamento no adolescente, pretendendo contribuir com subsídios para a melhoria do conhecimento dos profissionais (de saúde e em escolas) que trabalham com adolescentes e seus pais. Metodologia: A qualidade das atitudes dos pais foi considerada a partir da intensidade das perceções do afeto e das práticas parentais, enquanto a esfera comportamental incluiu comportamentos, de natureza internalizada e externalizada, reportados pelos adolescentes sobre as suas próprias ações e sentimentos. 0 protocolo de recolha de dados é composto por um questionário de caracterização sociodemográfica (tendo em consideração variáveis demográficas como a idade, sexo, localidade de residência, reprovações, coabitação e nível socioeconómico), pela adaptação portuguesa do Youth Self-Report (YSR) e pelo Inventário de Perceções adolescentes (IPA) adaptado por Fleming em 1997. A amostra incluiu 409 adolescentes de ambos os sexos, com idades compreendidas entre os 12 e os 19 anos ( $(M=14.93)$, de várias escolas e instituiç̧ões dos distritos de Portalegre e de Évora. Resultados: Os resultados sugerem, de um modo geral, que as perceções positivas dos adolescentes sobre as atitudes parentais se relacionam inversamente com a manifestação de problemas de comportamento nas suas duas dimensões, internalizados e externalizados. Foram encontradas diferenças significativas em função da idade e do sexo. Conclusões: São referidas implicações em termos de intervenção dos profissionais com os adolescentes e principalmente com os seus pais.
\end{abstract}

Palavras-chave: atitudes parentais; adolescência; problemas de comportamento internalizado; problemas de comportamento externalizado; intervenção. 


\section{ABSTRACT}

Parental attitudes' perceptions and adolescents behaviour problems. Introduction: The role of parents' educational attitudes in adolescent's development and behaviour has been broadly supported, emerging an interest for research in this field, not only as protector factor but also as risk factor for some deviance and mental health problems. The present investigation was designed to analyse the association between the parental attitudes' perceptions and the expression of adolescent behaviour problems, aiming to contribute with subsidies to the improvement of knowledge of professionals (health and schools) who work with adolescents and their parents. Methodology: The parents' attitudes quality was addressed considering the intensity of perceptions of affect and parental practices, while the behavioural problems include internalizing and externalizing behaviours, reported by the adolescents about their own actions and feelings. The data collection protocol is composed by a sociodemographic questionnaire (e.g., age, sex, place of residence, school failure, cohabitation and socioeconomic level), the Portuguese adaptation of the Youth Self-Report (YSR), and Fleming's (1997) Adolescents' Perceptions Inventory (IPA). The sample included 409 adolescents of both sexes, with ages ranging from 12 to 19 years old $(\mathrm{M}=14.93)$, from schools and other youth institutions, in the Portalegre and Évora districts. Results: Results suggest, in general, that adolescents' positive perceptions of parental attitudes have an inverse relation with the expression of internalizing and externalizing problems. Significant differences were found in function of age and sex. Conclusions: Implications are referred in terms of professionals' intervention with adolescents and mainly with their parents.

Keywords: parental attitudes; adolescence; internalizing problems; externalizing problems; intervention.

\section{INTRODUÇÃO}

0 papel desempenhado pelas atitudes educativas parentais no desenvolvimento e comportamento na adolescência tem sido amplamente suportado pela investigação, surgindo um interesse pela sua pesquisa não só enquanto fator protetor, mas também como fator de risco para alguns desvios e problemas de saúde mental (e.g., Roberts, Andrews, Lewinsohn \& Hops, 1990, cit. em Fanti, Henrich, Brookmeyer \& Kuperminc, 2008). Os sintomas internalizados e externalizados estão entre os problemas de saúde mental mais preocupantes na adolescência (Costello et al., 2011, cit. in Leadbeater \& Homel, 2015) e, embora os problemas externalizados tipicamente diminuam com a idade, os internalizados demonstram considerável estabilidade ou crescimento durante essa etapa; além disso, a coocorrência entre uma variedade de sintomas internalizados e externalizados permanece robusta (por exemplo, Leadbeater et al., 2012, cit. in Leadbeater \& Homel, 2015), sendo necessária uma atenção especial a fatores que se mostrem relevantes, quer para o risco quer para a prevenção dos problemas de comportamento e de saúde mental.

\section{OBJETIVOS DA INVESTIGAÇÃO}

Esta investigação pretende:

1) analisar a associação entre as perceções das atitudes parentais e a manifestação de problemas de comportamento no adolescente (de natureza exteriorizada e natureza interiorizada);

2) compreender se variáveis como o sexo e a idade desempenham um papel significativo no desenvolvimento de problemas de comportamento e se influenciam a perceção que 0 adolescente tem das atitudes parentais;

3) contribuir, através dos resultados alcançados, com subsídios para a melhoria do conhecimento dos profissionais (de saúde e em escolas) que trabalham com adolescentes e seus pais. 


\section{PSICOLOGÍA, INFANCIA Y EDUCACIÓN}

\section{AMOSTRA}

Para este estudo foi selecionada uma amostra de adolescentes na Região do Alentejo, distritos de Portalegre e Évora, com idades compreendidas entre os 12 e os 19 anos, de ambos os sexos, a frequentar 0 ensino regular ou profissional em escolaridade obrigatória, do $7^{0}$ ao $12^{\circ}$ ano. Trata-se de uma amostragem não probabilística e ocasional. Houve, no entanto, a preocupação de diversificar o local da recolha o mais possível, tentando abranger uma população com características diversas.

A amostra é constituída por 409 indivíduos, sendo que 50.1\% são do sexo feminino e $49.9 \%$ são do sexo masculino. Na Tabela 1 é possível conhecer a distribuição da amostra pela instituição (escolas e outras), idade, sexo, nacionalidade e localidade (rural ou urbana).

A média de idade dos adolescentes foi de 14.93 anos (DP =1.838). A recolha de dados foi realizada, maioritariamente, em sala de aula, excetuando quatro situações, em que a recolha se realizou nas instalações de outras instituições de caráter recreativo.

Em relação à composição do agregado familiar dos sujeitos da amostra foram codificadas oito variáveis, sendo de explicitar: a "Família Reconstruída" como incluindo a coabitação com pai/madrasta e mãe/padrasto; e "Nenhum Progenitor", quando o indivíduo vive com outros familiares como os avós, irmãos adultos ou tios, sem presença dos pais. Verifica-se que a maioria dos adolescentes coabita com a família nuclear intacta $(71.9 \%)$ e que a existência de famílias sem qualquer progenitor é muito reduzida (3.2\%) (Tabela 2).

Na sua grande maioria, os agregados familiares incluem-se no nível socioeconómico baixo (47.9\%), não existindo grande discrepância entre a frequência deste e a do nível socioeconómico médio (41.1\%). Apenas 10 famílias (2,4\%) reuniram características para o nível socioeconómico elevado, sendo que 0 nível de 8,6\% das famílias não foi especificado. 
Tabela 1 - Distribuição da amostra pela instituição, idade, sexo, nacionalidade e localidade (rural ou urbana) $(N=409)$.

\begin{tabular}{|c|c|c|c|}
\hline & & $\mathrm{n}$ & $\%$ \\
\hline \multirow[t]{9}{*}{ Instituição } & Grupo de Dança do CFEstremoz & 4 & $1.0 \%$ \\
\hline & E. P. de Alter do Chão & 31 & $7.6 \%$ \\
\hline & Sport Clube Borbense & 20 & $4.9 \%$ \\
\hline & Escuteiros de Vila Viçosa & 7 & $1.7 \%$ \\
\hline & E. S. $/ 3$ de Estremoz & 106 & $25.9 \%$ \\
\hline & E. B. de Sousel & 53 & $13.0 \%$ \\
\hline & Grupo de Dança “Tanzer” & 4 & $1.0 \%$ \\
\hline & E. B. de Portalegre & 76 & $18.6 \%$ \\
\hline & E. S. $/ 3$ de Portalegre & 108 & $26.4 \%$ \\
\hline \multirow[t]{8}{*}{ Idade } & 12 anos & 32 & $7.8 \%$ \\
\hline & 13 anos & 64 & $15.6 \%$ \\
\hline & 14 anos & 89 & $21.8 \%$ \\
\hline & 15 anos & 88 & $21.5 \%$ \\
\hline & 16 anos & 56 & $13.7 \%$ \\
\hline & 17 anos & 28 & $6.8 \%$ \\
\hline & 18 anos & 36 & $8.8 \%$ \\
\hline & 19 anos & 16 & $3.9 \%$ \\
\hline \multirow[t]{2}{*}{ Sexo } & Feminino & 205 & $50.1 \%$ \\
\hline & Masculino & 204 & $49.9 \%$ \\
\hline \multirow[t]{2}{*}{ Nacionalidade } & Portuguesa & 400 & $97.8 \%$ \\
\hline & Outra & 9 & $2.2 \%$ \\
\hline \multirow[t]{2}{*}{ Localidade } & Rural & 268 & $65.5 \%$ \\
\hline & Urbana & 141 & $34.5 \%$ \\
\hline
\end{tabular}


PSICOLOGÍA, INFANCIA Y EDUCACIÓN

Tabela 2 - Distribuição da amostra segundo o tipo de agregado familiar, considerando os elementos em coabitação $(N=409)$

\begin{tabular}{lccc}
\hline & $\mathrm{n}$ & $\%$ \\
\hline Coabitação & Mãe e Pai & 294 & $71.9 \%$ \\
Mãe, Pai e Avós / Tios & 20 & $4.9 \%$ \\
Só Mãe & 41 & $10.0 \%$ \\
Mãe e Avós / Tios & 5 & $1.2 \%$ \\
Só Pai & 9 & $2.2 \%$ \\
Pai e Avós / Tios & 3 & $0.7 \%$ \\
Família Reconstruída & 24 & $5.9 \%$ \\
Nenhum Progenitor & 13 & $3.2 \%$ \\
\hline
\end{tabular}

\section{METODOLOGIA E INSTRUMENTOS}

A recolha de dados foi realizada através do preenchimento de questionários, após o consentimento informado. Para a participação dos menores de idade, foi obtida a autorização dos pais. Os adolescentes preencheram individualmente os seguintes instrumentos:

(1) Youth Self Report - YSR (Achenbach, 1991; adap. Portuguesa: Fonseca e Monteiro, 1999). É uma escala que tem como finalidade avaliar dimensões do comportamento a partir de um questionário de autorresposta sobre os próprios comportamentos. Nesta pesquisa foi utilizada a versão para indivíduos dos 11 aos 18 anos, que se divide em duas partes. A primeira parte é constituída por 17 itens relacionados com competências, atividades e interesses sociais do individuo. A segunda parte é composta por 119 itens, dos quais 103 se relacionam com problemas de comportamento e 16 com comportamentos socialmente desejáveis. 0 preenchimento da escala é realizado a partir da resposta a cada item com três opções possíveis, 0 (não verdadeira), 1 (às vezes verdadeiras) e 2 (muitas vezes verdadeira) e é relativa a comportamentos nos últimos 6 meses. A adaptação realizada por Fonseca e Monteiro (1999) apresenta valores satisfatórios de consistência interna, obtendo para o score total 0,80 e valores que variam entre 0,70 e 0,80 para as suas 6 dimensões e 2 clusters (ver na Tabela 3 as suas designações).

(2) Inventário de Perceções Adolescentes - IPA (Streit, 1978; adap. Portuguesa: Fleming, 1997). Tem como finalidade avaliar a perceção dos adolescentes em relação às atitudes parentais, ou seja, a perceção que 0 individuo tem da qualidade e da intensidade da relação que tem com os seus pais ou com quem os substitua. Foi validado para a população portuguesa utilizando uma amostra de indivíduos com idades compreendidas entres os 12 e os 19 anos. Esta escala é composta por 63 perguntas relativamente às atitudes dos progenitores, com quatro respostas possíveis: Mãe e Pai, Só Mãe, Só Pai, Nem Mãe nem Pai. 0 IPA divide-se em duas dimensões, nomeadamente, a dimensão do Afeto (Amor versus Hostilidade) e a dimensão das Práticas Educativas (Autonomia versus Controlo), remetendo a primeira para a qualidade afetiva da relação pais-filhos e a segunda para as práticas educativas usadas pelos pais. Na adaptação para a população portuguesa (Fleming, 1988, cit. in Fleming, 1997), o cálculo do coeficiente Alpha de Cronbach revelou valores de 0,93 na dimensão Amor, 0,79 na dimensão Hostilidade, 0,65 na dimensão Controlo e 0,53 na dimensão Autonomia.

(3) um Questionário Sociodemográfico, construído para o presente estudo, que teve como objetivo recolher dados sociodemográficos, nomeadamente, a idade, 0 sexo, a nacionalidade, a localidade de residência, 0 ano de 


\section{PERCEÇÃO DE ATITUDES PARENTAIS E PROBLEMAS DE COMPORTAMENTO NOS ADOLESCENTES}

escolaridade, a quantidade de reprovações, características do agregado familiar como a composição, as idades dos membros, as habilitações literárias, as profissões e a situação profissional.

Na análise de dados, para compreender a distribuição e a homogeneidade da variância dos dados recolhidos, foram calculados o teste de Kolmogorov-Smirnov e o teste de Levene. 0 teste de Kolmogorov-Smirnov revelou um valor de $p<0.05$, tanto para as distribuições globais das escalas, como para as distribuições pelos diversos grupos a explorar. A partir destes resultados, podemos concluir que os dados não seguem uma distribuição normal. 0 teste de Levene revelou variâncias homogéneas, com $p>0.05$. Violado o principal pressuposto para utilizar testes paramétricos, optou-se pelos testes não paramétricos.

Assim, para as análises estatísticas, recorreu-se ainda ao coeficiente de correlação ró de Spearman para explorar a intensidade e direção da associação entre duas variáveis; e utilizou-se o teste U de Mann-Whitney para comparar dois grupos independentes.

\section{RESULTADOS}

1) Relação entre a perceção das atitudes parentais e a manifestação de problemas de comportamento

Partindo da hipótese de que uma perceção positiva das atitudes dos pais (Amor e Autonomia) se relaciona com uma diminuição de Problemas de Comportamento e vice-versa, procedeu-se ao cálculo do coeficiente de correlação ró de Spearman.

Verificou-se, então, que as dimensões positivas das atitudes parentais (Amor e Autonomia) revelaram correlações significativas negativas e pequenas com os fatores da escala de Problemas de Comportamento, destacando-se a da dimensão de Amor ( $r o ́=-0.391, p<0.01, n=409$ ) com o Score Total de Comportamento, assim como a da Autonomia com 0 fator de Problemas de Atenção/Hiperatividade (ró $=-0.161, p<0.01, n=409$ ). A dimensão Hostilidade obteve correlações significativas positivas e pequenas com todos os fatores, sendo a mais elevada (ró = 0.321, $p<0.01, n=409$ ) com a do Score Total de Comportamento. A dimensão Controlo não revelou correlações significativas com nenhum aspeto do comportamento da escala YSR.

2) Efeito da idade na perceção das atitudes parentais e na manifestação de problemas de comportamento

De acordo com a literatura, considerou-se que os adolescentes da classe etária dos 16 aos 19 anos tendem a apresentar valores mais elevados de perceção da dimensão Autonomia; e os dos 12 aos 15 anos tendem a apresentar valores mais elevados de perceção da dimensão Amor. Quanto à idade e à manifestação de problemas de comportamento, partiu-se da hipótese de que os adolescentes da classe etária dos 16 aos 19 anos tendem a apresentar valores mais elevados de manifestação de Problemas de Comportamento.

Para analisar a contribuição da idade em relação à perceção das atitudes parentais e à manifestação de problemas de comportamento, recorreu-se ao teste $U$ de Mann-Whitney. A variável idade dos sujeitos foi codificada em duas classes etárias, dos 12 aos 15 anos e dos 16 aos 19 anos. Esta classificação foi elaborada para distinguir fases específicas da adolescência, usualmente apontadas na literatura sobretudo no que se refere à evolução do comportamento desviante (Morizot \& Kazemian, 2015; Morgado \& Vale Dias, 2015). Os resultados obtidos evidenciam cinco diferenças entre classes consideradas significativas, três delas a um nível de significância $p<0.01$ e duas com valores de $p<0.05$ (Tabela 3). 
Tabela 3 - Médias, desvios-padrão e teste U de Mann-Whitney das escalas YSR e IPA em função da classe etária.

\begin{tabular}{lcccccc}
\hline & \multicolumn{2}{c}{$\begin{array}{c}12-15 \\
(\mathrm{~N}=273)\end{array}$} & \multicolumn{2}{c}{$16-19$} & $\mathrm{U}$ & $\mathrm{p}$ \\
\cline { 1 - 5 } & $\mathrm{M}$ & $\mathrm{DP}$ & $\mathrm{M}$ & $\mathrm{DP}$ & & \\
\cline { 2 - 5 } IPA_Amor & 146.26 & 39.41 & 132.62 & 47.25 & 15588.000 & $0.008^{* *}$ \\
IPA_Hostilidade & 42.15 & 32.68 & 48.14 & 36.62 & 16554.500 & 0.074 \\
IPA_Autonomia & 114.71 & 45.76 & 127.57 & 45.38 & 15493.000 & $0.006^{* *}$ \\
IPA_Controlo & 123.88 & 39.45 & 122.06 & 43.65 & 18557.500 & 0.995 \\
YSR_Comport. Antissocial & 3.12 & 2.96 & 3.87 & 3.05 & 15530.000 & $0.007^{* *}$ \\
YSR_Atenção/Hiperatividade & 7.66 & 4.38 & 7.54 & 3.84 & 18392.000 & 0.878 \\
YSR_Ansiedade/Depressão & 3.05 & 3.59 & 3.43 & 3.47 & 16672.000 & 0.089 \\
YSR_Isolamento & 6.08 & 3.13 & 6.58 & 2.87 & 16626.000 & 0.084 \\
YSR_Queixas Somáticas & 2.61 & 2.40 & 2.84 & 2.83 & 18261.000 & 0.785 \\
YSR_Problemas Pensamento & 2.11 & 2.36 & 2.47 & 2.42 & 16564.000 & 0.070 \\
YSR_Total & 38.31 & 20.18 & 41.78 & 20.24 & 16351.000 & $0.049^{*}$ \\
YSR_Cluster Exteriorizado & 10.35 & 6.40 & 11.10 & 5.76 & 16614.500 & 0.083 \\
YSR_Cluster Interiorizado & 11.74 & 7.73 & 12.85 & 7.31 & 16326.500 & $0.047^{*}$ \\
\hline
\end{tabular}

Nota. ${ }^{*} p<0.01 ;{ }^{*} p<0.05$

É de assinalar a diferença entre idades na perceção das dimensões Amor e Autonomia, com uma média mais alta na classe etária dos $12-15$ anos para a primeira ( $M=146.26$ vs. $M=132.62)$ e uma média mais elevada na classe etária dos $16-19$ anos para a segunda ( $M=127.57$ vs. $M=114.71$ ). Conclui-se que enquanto a classe etária mais nova apresenta valores de perceção das atitudes parentais mais elevados no Amor, os indivíduos mais velhos do estudo apresentam perceções mais elevadas na Autonomia.

Em relação às diferenças na escala de problemas de comportamento, apenas um dos fatores se destacou sozinho, o Comportamento Antissocial, no qual a classe etária mais velha apresentou valores mais elevados ( $\mathrm{M}$ $=3.87$ vs. $M$ =3.12). 0 score global e 0 cluster Interiorizado apresentaram também valores significativos, ambos com resultados médios mais elevados na classe etária dos 16 aos 19 anos. Estes resultados revelam uma maior tendência para a manifestação de comportamentos antissociais em idades entre os 16 e 19 anos, tal como valores mais elevados de sintomatologia de psicopatologia e de problemas de natureza internalizada.

3) Efeito do sexo na perceção das atitudes parentais e na manifestação de problemas de comportamento A comparação entre rapazes e raparigas nas escalas YSR e IPA encontra-se na Tabela 4. 
Tabela 4 - Médias, desvios-padrão e teste U de Mann-Whitney das escalas YSR e IPA em função do sexo

\begin{tabular}{lcccccc}
\hline & \multicolumn{2}{c}{$\begin{array}{c}\text { Feminino } \\
(\mathrm{N}=205)\end{array}$} & \multicolumn{2}{c}{$\begin{array}{c}\text { Masculino } \\
(\mathrm{N}=204)\end{array}$} & $\mathrm{U}$ & $\mathrm{p}$ \\
\cline { 1 - 5 } & $\mathrm{M}$ & $\mathrm{DP}$ & $\mathrm{M}$ & $\mathrm{DP}$ & & \\
\cline { 2 - 5 } IPA_Amor & 138.68 & 41.21 & 144.79 & 43.86 & 18635.500 & 0.057 \\
IPA_Hostilidade & 43.07 & 29.83 & 45.21 & 37.98 & 20483.000 & 0.720 \\
IPA_Autonomia & 114.23 & 44.52 & 123.77 & 47.02 & 18243.500 & $0.025^{*}$ \\
IPA_Controlo & 118.93 & 36.59 & 127.65 & 44.38 & 17927.500 & $0.012^{*}$ \\
YSR_Comport. Antissocial & 2.98 & 2.84 & 3.76 & 3.12 & 17408.000 & $0.003^{* *}$ \\
YSR_Atenção/Hiperatividade & 8.10 & 4.29 & 7.14 & 4.07 & 18108.000 & $0.019^{*}$ \\
YSR_Ansiedade/Depressão & 3.94 & 3.85 & 2.41 & 3.05 & 15068.500 & $0.000^{* *}$ \\
YSR_Isolamento & 7.04 & 3.08 & 5.45 & 2.81 & 14560.500 & $0.000^{* *}$ \\
YSR_Queixas Somáticas & 3.28 & 2.54 & 2.09 & 2.43 & 14058.00 & $0.000^{* *}$ \\
YSR_Problemas Pensamento & 2.55 & 2.66 & 1.91 & 2.02 & 18369.500 & $0.030^{*}$ \\
YSR_Total & 42.60 & 20.86 & 36.31 & 19.14 & 16828.000 & $0.001^{* *}$ \\
YSR_Cluster Exteriorizado & 10.57 & 6.14 & 10.63 & 6.27 & 20890.000 & 0.987 \\
YSR_Cluster Interiorizado & 14.26 & 7.95 & 9.95 & 6.57 & 13504.000 & $0.000^{* *}$ \\
\hline
\end{tabular}

Nota. ${ }^{* *} p<0.01 ;{ }^{*} p<0.05$

Os resultados revelam grande expressividade nas diferenças entre os sexos relativas a todos os fatores do comportamento, nos quais os valores médios do sexo masculino superaram os do sexo feminino em apenas um fator, 0 do Comportamento Antissocial ( $M=3.76$ vs. $M=2.98)$. Nos restantes cinco fatores a média do sexo feminino ultrapassa sempre a do sexo oposto (Tabela 4). 0 Total da escala de comportamento e o cluster Interiorizado também obtiveram valores com significância estatística, com 0 sexo feminino a liderar nos valores médios de ambos, $M_{F}=42.62$ vs. $M_{M}=36.31$ para o primeiro e $M_{F}=14.26$ vs. $M_{M}=9.95$ para 0 segundo. Assim, 0 sexo feminino manifesta valores médios mais elevados em quase todos os problemas de comportamento, com destaque para a maior significância nos problemas incluídos no cluster de problemas interiorizados e para o valor total de psicopatologia (Total da escala). Como já referido, o sexo masculino destaca-se apenas pelos valores mais elevados no comportamento antissocial, um dos exteriorizados.

Em relação à escala de perceções de atitudes parentais, os resultados evidenciam valores significativamente diferentes em duas dimensões, Autonomia $(U=18243.5, p=0.025)$ e Controlo $(U=17927.5, p=0.012)$, com 0 sexo masculino a percecionar valores médios mais altos em ambos.

\section{DISCUSSÃO}

A presente investigação apoia a hipótese de que uma perceção positiva das atitudes dos pais (Amor e Autonomia) se relaciona com uma diminuição de Problemas de Comportamento e vice-versa. A relação inversa ou negativa entre a educação parental positiva (pela conjugação de Amor com Autonomia) e o comportamento desviante apoia os resultados obtidos em estudos anteriores (e.g., Vale Dias, Martinho, Franco-Borges \& VazRebelo, 2012). Este resultado sugere que uma maior autonomia percecionada se associa a uma menor manifes- 
tação de comportamentos negativos, comprovando a importância da promoção de uma autonomia adequada na adolescência (e.g., Relvas, 2000) e a sua centralidade neste período como tarefa desenvolvimental moderadora (e.g., Steinberg \& Silk, 2002; Zimmer-Gembeck \& Collins, 2003, cit. in Smetana, 2012). Os dados indicam que a perceção de maior disponibilidade emocional, suporte, amor, coesão e afeto parental se associam à diminuiç̧ão de comportamentos desviantes de natureza internalizada e externalizada, contrariamente a práticas parentais caracterizadas por hostilidade, criticismo, coerções agressivas e rejeição parental, comprovando estudos anteriores (e.g., White \& Renk, 2012; Buehler, 2006; Juang \& Silbereisen, 1999, cit. in Bonino, Cattelino \& Ciairano, 2005).

Foi ainda possível perceber que os adolescentes da classe etária dos 16 aos 19 anos tendem a apresentar valores mais elevados de perceção da dimensão Autonomia e que os dos 12 aos 15 anos tendem a apresentar valores mais elevados de perceção da dimensão Amor. Os resultados corroboram investigações de vários autores que associam uma maior perceção de autonomia (e uma menor perceção de controlo) aos adolescentes mais velhos (e.g., Fleming, 1988, cit. In Fleming, 1997; Goede, Branje \& Meeus, 2009; Ducharne et al., 2006, cit. in Soares \& Almeida, 2011). Este aumento da perceção de Autonomia com a idade parece associar-se ao facto de, nas sociedades ocidentais, os pais tenderem a dar mais liberdade aos filhos à medida que vão crescendo (e.g., Oliveira, 1994). Merece ainda referência a crescente maturação cognitiva que capacita 0 adolescente para compreender melhor as situações sociais, contribuindo para a possibilidade de the ser fornecida uma maior liberdade de ação. Quanto à dimensão Amor, os resultados corroboram conclusões obtidas noutros estudos que associam uma maior perceção de amor e suporte parental à fase inicial da adolescência (e.g., Fleming, 1988; Meus et al., 2005, cit. in Soares \& Almeida, 2011). A possível causa do decréscimo da perceção de Amor numa fase mais avançada da adolescência pode prender-se com maior necessidade de privacidade do adolescente, surgindo com esta necessidade um maior afastamento, como também uma diminuição de expressão de afetos físicos entre pais e filhos; no entanto, este decréscimo pode não se relacionar com uma perda de amor ou de respeito entre ambos (e.g., Montemayor, 1983, 1986, cit. in Steinberg \& Silk, 2002).

Nos adolescentes mais velhos verificaram-se valores mais elevados de manifestação de Problemas de Comportamento, o que parece comprovar investigações anteriores que colocam o pico de prevalência do comportamento desviante nesta classe etária, principalmente quando nos referimos a comportamentos antissociais (e.g., Morizot \& Kazemian, 2015, Morgado \& Vale Dias, 2013, 2015; Vale Dias et al., 2015), não deixando de se verificar também em comportamentos internalizados (e.g., Hankin et al., 1998, cit. in O'Mara, Lee \& King, 2013).

Quanto ao efeito do sexo na perceção das atitudes parentais, os resultados revelaram o sexo masculino a percecionar valores mais elevados em ambas as dimensões Autonomia e Controlo. Estes resultados contrariam a ideia de que o sexo feminino perceciona necessariamente mais controlo (e.g., Ducharne et al., 2006, cit. in Soares \& Almeida, 2011; Oliveira, 1994), havendo já estudos que se direcionam no mesmo sentido que o presente, com o sexo masculino a percecionar mais autonomia mas também mais controlo (e.g., Maia, 2014). Sugere-se, futuramente, realizar análises mais profundas, que incluam a diferenciação por idades e a avaliação de possíveis alterações de práticas educativas relacionadas com o género.

Ainda foi possível verificar que, em relação aos problemas de comportamento, o sexo feminino se apresentou com valores mais elevados em todas as dimensões de Comportamento Internalizado ( $U=13504, p=0.000)$, enquanto o sexo masculino apenas apresentou valores mais elevados num dos fatores externalizados, na dimensão do Comportamento Antissocial $(U=17408, p=0.003)$, corroborando uma vasta bibliografia sobre 0 tema (e.g., Storvoll, Wichstrom \& Pape, 2003, cit. in Gavazzi, 2011; Berkout, Young \& Gross, 2011; Moffitt, 2006; Tremblay, 2010, cit. in Vale Dias et al., 2015; Morgado \& Vale Dias, 2015). No entanto, 0 sexo feminino indicou também a média mais elevada nos Problemas de hiperatividade/atenção e no Total da escala, sendo que esta evidência pode ir no sentido de que, embora o sexo masculino apresente um padrão mais comum de comportamentos de natureza externalizada, a evolução dos mesmos problemas nas raparigas também pode ser preocupante (e.g., Pacheco et al., 2005). 


\section{PERCEÇÃO DE ATITUDES PARENTAIS E PROBLEMAS DE COMPORTAMENTO NOS ADOLESCENTES}

\section{CONCLUSÕES}

Os resultados sugerem, de um modo geral, que as perceções positivas dos adolescentes sobre as atitudes parentais se relacionam inversamente com a manifestação de problemas de comportamento nas suas duas dimensões, internalizados e externalizados. Esta evidência corrobora estudos que consideram, no desenvolvimento de problemas internalizados e externalizados, 0 efeito de contínuas influências entre 0 adolescente e 0 seu ambiente familiar (e.g., Fanti et al., 2008). Esta pesquisa revela ainda diferenças significativas, em função da idade e do sexo, quer a nível da perceção das atitudes parentais quer em termos de comportamento dos adolescentes. 0 estudo destas diferenças deverá continuar a ser aprofundado e as suas especificidades devem ser consideradas na intervenção.

Como implicações dos resultados deste estudo, sugere-se que uma prevenção comportamental em adolescentes não pode, nem deve, ser introduzida sem incluir o sistema familiar, dado que este se demonstra associado ao comportamento relatado pelos adolescentes na presente pesquisa, podendo ser visto como um moderador importante na manifestação de comportamentos não adaptativos, noção apoiada por extensa bibliografia sobre a temática (e.g., Lipsey \& Derzon, 1998; Chamberlain, 1999; Tremblay et al., 2000, cit. in Fonseca, 2002; Hussey et al., 2006, cit. in Chapin \& Coleman, 2014; Mack, Peck, \& Lieber, 2015; Mendes, \& Vale-Dias, 2018). Finalmente, refere-se, ainda, a necessidade de continuar a investir no desenvolvimento de atitudes parentais positivas, pautadas pelo amor e autonomia, dada a sua relação com o comportamento equilibrado dos filhos.

\section{REFERÊNCIAS BIBLIOGRÁFICAS}

Achenbach, T. M. (1991). Manual for the youth self-report and 1991 profile. Burlington, VT: Department of Psychiatry, University of Vermont.

Bonino, S., Cattelino, E., \& Ciairano, S. (2005). Adolescents and risk: behaviors, functions and protective factors. Itália: Springer.

Buehler, C. (2006). Parents and peers in relation to early adolescent problem behavior. Journal of Marriage and Family, 68, 109-124. doi: 10.1111/j.1741-3737.2006.00237.x

Chapin, J., \& Coleman, G. (2014). Adolescents' perceptions of family violence risks. Journal of Family Violence, 29, 757-761. doi: 10.1007/s10896-014-9634-1

Fanti, K. A., Henrich, C. C., Brookmeyer, K. A., \& Kuperminc, G. P. (2008). Toward a transactional model of parentadolescent relationship quality and adolescent psychological adjustment. The Journal of Early Adolescence, 28(2), 252-276. doi: 10.1177/0272431607312766

Fleming, M. (1997). Adolescência e autonomia: o desenvolvimento psicológico e a relação com os pais. Porto: Edições Afrontamento.

Fonseca, A. C., \& Monteiro, C. M. (1999). Um inventário de problemas do comportamento para crianças e adolescentes: 0 Youth Self-Report de Achenbach. Psychologica, 21, 79-96.

Gavazzi, S. M. (2011). Families with adolescents: bridging the gaps between theory, research, and practice. New York: Springer.

Leadbeater, B. J., \& Homel, J. (2015). Irritable and defiant sub-dimensions of ODD: their stability and prediction of internalizing symptoms and conduct problems from adolescence to young adulthood. Journal of Abnormal Child Psychology, 43, 407-421. doi: 10.1007/s10802-014-9908-3

Mack, K. Y., Peck, J. H., \& Lieber, M. J. (2015). The effects of family structure and family processes on externalizing and internalizing behaviors of male and female youth: a longitudinal examination. Deviant Behavior, 36, 740-764. doi: 10.1080/01639625.2014.977117

Maia, A. (2014). Atitudes educativas parentais, resiliência e rendimento académico do adolescente: análise de relações e contributo de variáveis demográficas. Dissertação de Mestrado apresentada à Faculdade de Psicologia e de Ciências de Educação da Universidade de Coimbra.

Mendes, L. S., \& Vale-Dias, M. L. (2018). Relação entre estilos educativos parentais, confiança interpessoal e 
vinculação na adolescência. Psicologia, Saúde \& Doenças, 19(1), 136-143. https://dx.doi.org/10.15309/18psd190120

Morgado, A. M., \& Vale Dias, M. L. (2013). The antisocial phenomenon in adolescence: what is literature telling us? Aggression and Violent Behavior, 18, 436-443. doi: 10.1016/j.avb.2013.05.004

Morgado, A. M., \& Vale Dias, M. L. (2015). Adolescent antisocial behaviour: a development approach to individual perceptions. Paper presented at the 17th ECDP - September 8-12, Braga, Portugal.

Morizot, J., \& Kazemian, L. (2015). Introduction: understanding criminal and antisocial behavior within a developmental and multidisciplinary perspective. In J. Morizot \& L. Kazemian. (Eds.), The development of criminal and antisocial behaviour: theory, research and practical applications (1-18). New York: Springer.

Oliveira, J. H. (1994). Psicologia da educação familiar. Coimbra: Almedina.

O'Mara, R. M., Lee, A., \& King, C. A. (2013). Depression and suicide-related behaviors in adolescence. In W. T. O'Donohue, L. T. Benuto, \& L. W. Tolle. (Eds.), Handbook of adolescent health psychology (521-535). New York: Springer.

Pacheco, J., Alvarenga, P., Reppold, C., Piccinini, C. A., \& Hutz, C. S. (2005). Estabilidade do comportamento anti-social na transição da infância para a adolescência: uma perspectiva desenvolvimentista. Psicologia: Reflexão e Crítica, 18 (1), 55-61. doi: http://dx.doi.org/10.1590/S0102-79722005000100008.

Relvas, A. (2000). Adolescente(s), família(s) e escola(s). In M. T. Medeiros, \& A. I. Serpa. (Eds.), Adolescência: abordagens, investigacoes e contextos de desenvolvimento (48-77). Lisboa: Direção Regional da Educação.

Soares, D. L., \& Almeida, L. S. (2011). Percepção dos estilos educativos parentais: sua variação ao longo da adoIescência. Libro de Actas Do XI Congresso International Galego-Português de Psicopedagoxia, 4071-4083.

Smetana, J. G. (2012). The role of trust in adolescent- parent relationships: to trust you is to tell you. In K. J. Rotenberg. (Ed.), Interpersonal trust during childhood and adolescence (223-246). Cambridge: Cambridge University Press.

Steinberg, L., \& Silk, J. S. (2002). Parenting adolescents. In M. H. Borstein. (Ed.), Handbook of parenting (103134). Mahwah, NJ: Lawrence Erlbaum Associates.

Streit, F. (1978). Technical manual: Youth Perception Inventory. Fred Streit Associates.

Vale Dias, M. L., Fonseca, A., Franco-Borges, G., Vaz-Rebelo, P., Oliveira, M., \& Moreira, A., \& De Man, J. (2015). Gender differences in strain, negative emotions, delinquency, substance use: implications for the general strain theory. Paper Presented at the 17th ECDP - September 8-12, Braga, Portugal.

Vale Dias, M. L., Martinho, L., Franco-Borges, G., \& Vaz-Rebelo, P. (2012). Estilos educativos parentais e comportamento antissocial na adolescência. International Journal of Developmental and Educational Psychology, 1 (1), 469-478. http://www.redalyc.org/pdt/3498/349832342048.pdf

White, R., \& Renk, K. (2012). Externalizing behavior problems during adolescence: an ecological perspective. Journal of Child and Family Studies, 21, 158-171. doi: 10.1007/s10826-011-9459-y 
